34th Midwest Symposium on Circuits and Systems; Monterey CA, 14-17 May 1991, pp.371-375.

Copyright (C) 1991 IEEE (DOI 10.1109/MWSCAS.1991.252174).

\title{
TABLE-DRIVEN ANALYSES IN THE SPICE-PAC CIRCUIT SIMULATION PACKAGE
}

\author{
W.M. Zuberek and M.S. Zuberek \\ Department of Computer Science \\ Memorial University of Newfoundland \\ St. John's, Canada A1C- 5 S7
}

\begin{abstract}
A b s t r a c t
Recent applications of SPICE-PAC to transistor parameter extraction and hierarchical simulation require rather flexible circuit analyses, performed for irregularly distributed values of independent variables. An implementation of table-driven analyses (DC, AC and time-domain) is described in which tables of arbitrarily distributed independent variables (voltages, frequencies or timepoints) are used rather than the fixed-step strategy implemented in SPICE-like simulators. Simple examples are used to illustrate applications of table-driven analyses.
\end{abstract}

\section{INTRODUCTION}

SPICE-PAC is a package of circuit simulation routines derived from the popular SPICE-2G circuit simulator from the University of California at Berkeley. It is upward compatible with SPICE, i.e., it accepts the same circuit description language and provides the same set of analyses, but it also contains a number of extensions and enhancements that are not available in SPICE; a hierarchical naming scheme, static and dynamic circuit variables, parameterized subcircuit expansions and elements of higher-level circuit specifications are a few examples of such enhancements. The most distinctive feature of SPICE-PAC is, however, its "open" structure, which means that all analyses as well as (dynamic) definitions of parameters and variables are performed "on demand", as required by specific applications. This also means that SPICE-PAC can easily be integrated with other CAD tools to provide circuit optimization, parameter extraction, statistical simulation, mixed-mode simulation, etc..

Recent applications of SPICE-PAC to transistor parameter extraction and hierarchical simulation (with tabledriven specifications of functional blocks) require rather flexible circuit analyses, performed for irregularly distributed values of independent variables. Since SPICElike simulators support fixed-step analyses only, the required flexibility could not be obtained without significant degradation of the simulation performance. Therefore the implementation of the three fundamental analyses, i.e., DC transfer curve analysis, frequency-domain (AC) analysis and time-domain (TR) analysis, were modified in order to allow specification of domains of independent variables by tables of arbitrarily distributed values rather than a fixed-step strategy. In parameter extraction, such tabledriven analyses must be performed at values (voltages, frequencies or timepoints) determined by measurement data; in table-driven specifications of functional blocks, variable spacing of data points is needed for accurate but efficient representation of nonlinearities.

The paper describes the extensions to the input language required for specification of table-driven analyses, and modifications of low-level interfaces for direct control of simulation in integrated applications; it also discusses changes in general organization of basic analyses. Simple examples of table-driven circuit simulation are used as illustrations.

\section{INPUT LANGUAGE EXTENSIONS}

For DC transfer curve, time-domain and frequencydomain analyses, table-driven options are described by appropriate extensions of the .DC, .TRAN and .AC directives, respectively. The original syntax of these directives is [Vlad]:

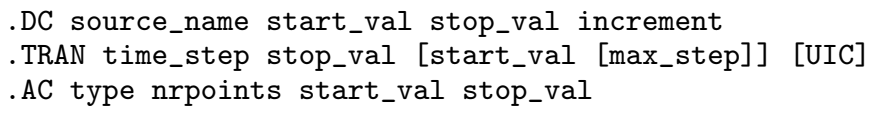

where lower-case phrases denote user-supplied names and/or values, upper-case fragments must be used verbatim (e.g., TRAN, UIC), and optional parts are enclosed in square brackets "[" and "]".

The table-driven specifications replace the "start", "stop" and "increment" values by a list of values, so the additional formats of these directives are:

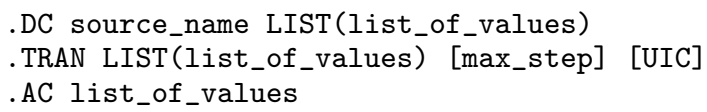

where list_of_values is a sequence of numbers (values of the independent voltage or current source indicated by source_name for the DC analysis, timepoints for TR analysis, or frequencies for $\mathrm{AC}$ analysis) separated by commas ",". For the time-domain analysis the values must be strictly increasing. For DC analysis the values can be specified in any order, however, preserving an increasing 
or decreasing order oif values improves efficiency of analysis, and may also improve convergence properties. For AC analysis, the ordering of values is insignificant.

The following simple example illustrates table-driven analyses:

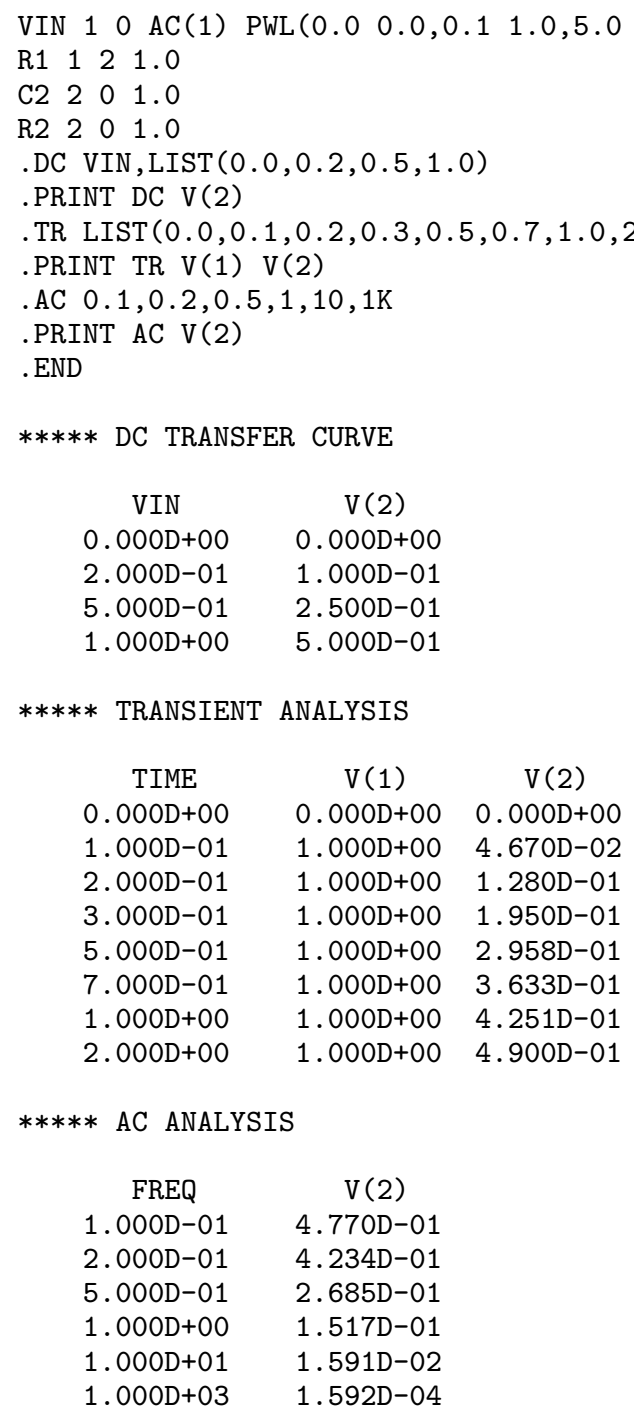

Table-driven analyses can also be indicated in the extended circuit description [Z1]; in this case the lists of values are enclosed in parentheses, as shown in the following example:

.END/EXT

.PAR/10 DCTC(VIN,$(0.0,0.1,0.5,0.9,1.0))$

.PAR/20 TR $((0.0,0.1,0.2,0.3,0.5,0.7,1.0,1.5,2.0), 0.1,0)$

$. P A R / 30 \operatorname{AC}((0.1,0.2,0.5,1.0,100.0))$

.END

\section{INTERNAL INTERFACES}

SPICE-PAC is an "open" simulation tool that performs simulation operations "on demand", as required by a specific application [Z1]. SPICE-PAC's operations (such as "define parameters for an analysis", "execute an analysis", etc.) are performed by invocations of corresponding interfacing routines with appropriate parameters [Z2]. Tabledriven analyses are indicated by modified invocations of the SPICED, SPICET and SPICEF routines.

\section{SPICED - define parameters for DC analysis}

The invocation of SPICED must be equivalent to the following Fortran statement [Z2]:

CALL SPICED (source,vistrt,vistop,nrstep,iflag

For table-driven DC analysis, vistrt is a DOUBLE PRECISION array that contains the list of values of an independent voltage or current source indicated by source. The vistop argument is immaterial in this case (although it must be DOUBLE PRECISION as well). nrstep must be set to the negative number of values in VISTRT, and iflg is the return flag, as in the original invocation.

\section{SPICET - define parameters for TR analysis}

The invocation of SPICET must be equivalent to the following Fortran statement [Z2]:

CALL SPICET (tmstrt,tmstop, nrstep, stpmax, incond,iflg)

For table-driven TR analysis, tmstrt is a DOUBLE PRECISION array that contains the list of required timepoints. The tmstop argument is immaterial in this case (although it must be DOUBLE PRECISION). nrstep must be set to the negative number of values in tmstrt, and the remaining arguments are the same as in the original invocation.

\section{SPICEF - define parameters for AC analysis}

The invocation of SPICEF must be equivalent to the following Fortran statement [Z2]:

CALL SPICEF (ittab,frtab,nrstep,iflg)

For table-driven AC analysis, frtab is a DOUBLE PRECISION array that contains the list of required frequencies. The ittab argument is immaterial in this case (although it must be INTEGER). nrstep must be set to the negative number of values in frtab, and iflg is the return flag, as in the original invocation.

\section{IMPLEMENTATION}

\section{DC transfer curve analysis}

The DC transfer curve analysis is basically a repetitive DC operating point solution performed for a range of values of one independent voltage or current source in the circuit; the only difference is in starting point which - for each new sweep point of DC analysis - is determined by a linear extrapolation from the previous points.

The general outline of this analysis is as follows [Coh] (VISRTR, VISTOP, VIINCR and SOURCE are DC analysis parameters, either defined by the .DC directive in the circuit description, or by an invocation of the SPICED routine): 


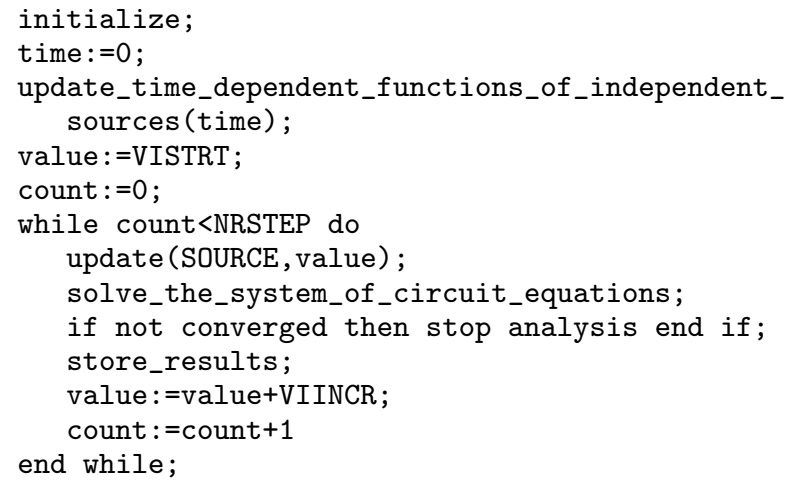

The modified version uses a logical variable DCLIST which is TRUE if the analysis is table-driven, otherwise it is FALSE; VITAB is an array that stores the list of values for a table-driven analysis:

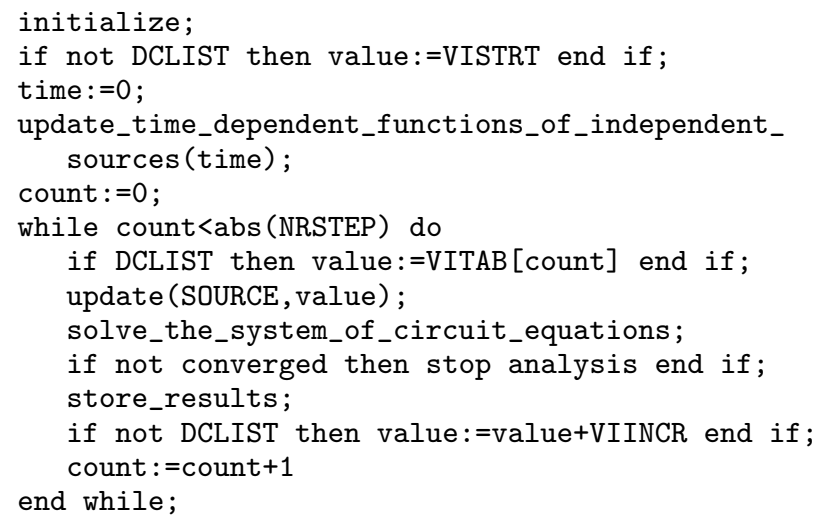

\section{Time-domain analysis}

The time-domain analysis is controlled by a variable timestep mechanism, so the actual timepoints selected for analysis are quite different than the "output timepoints" described in the parameters of this analysis. The (intermediate) solutions, obtained for internal timepoints, are simply stored in a temporary workspace, and in the final stage of analysis are used for linear interpolation of output timepoints. I order to use available workspace efficiently and speed up the analysis, intermediate results are stored for those timepoints only which are needed for final interpolation, i.e., those internal timepoints that "bracket" the output timepoints:

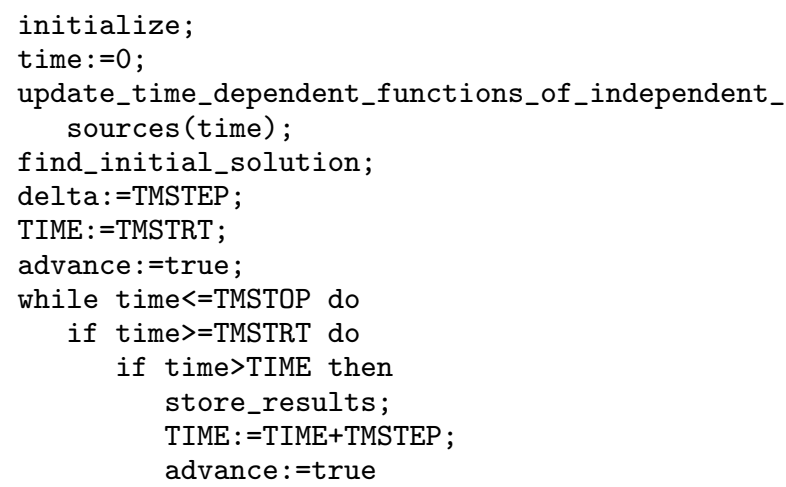

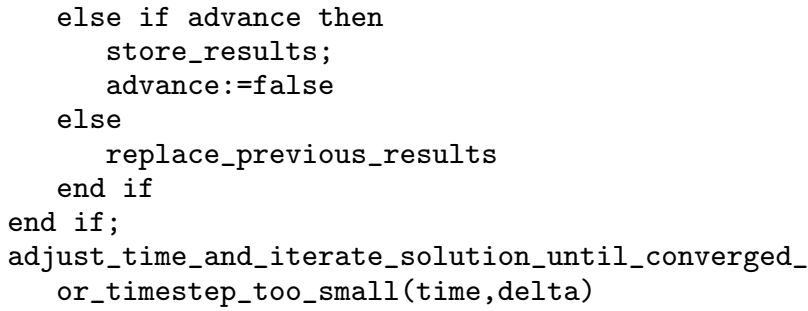

The modified version uses a logical variable TMLIST which is TRUE if the analysis is table-driven, otherwise it is FALSE, and an array TMTAB that stores the list of values for a table-driven analysis:

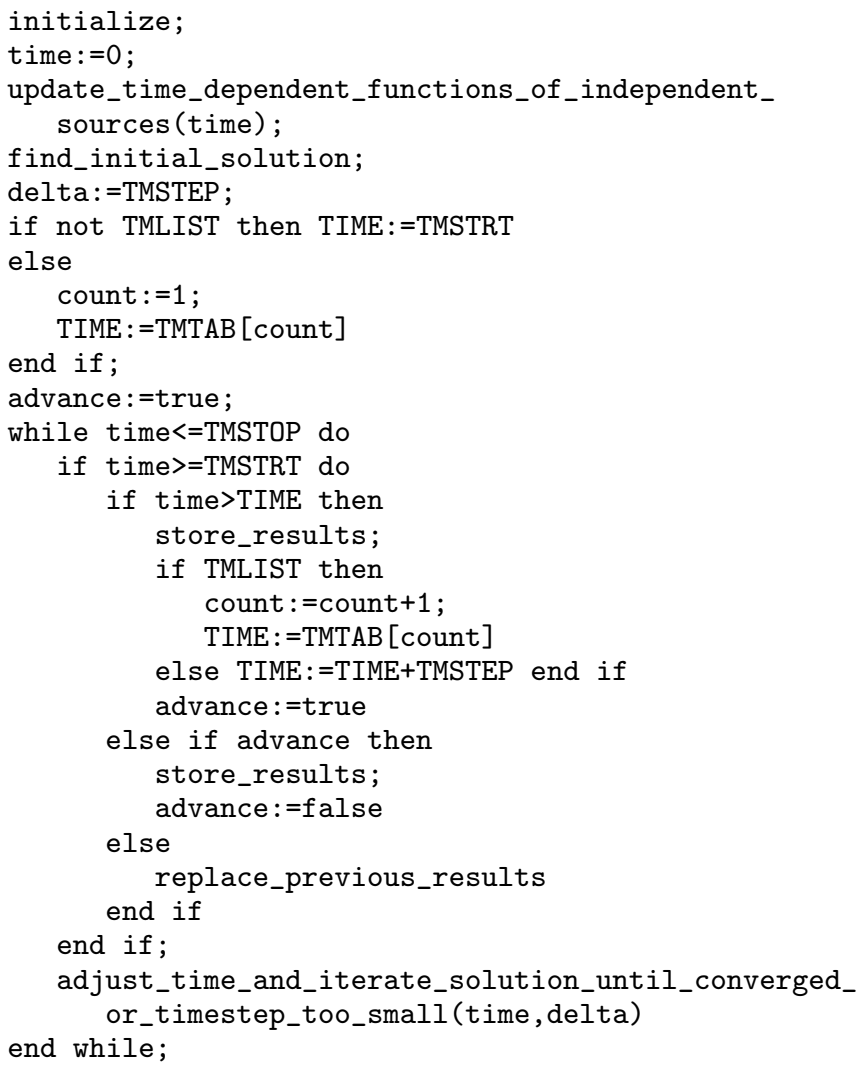

\section{Frequency-domain analysis}

The frequency-domain analysis can be performed with either linear or logarithmic variation; the actual choice (the type argument of the .AC line) is represented by a logical variable ACVAR which is TRUE for the linear variation and is FALSE otherwise:

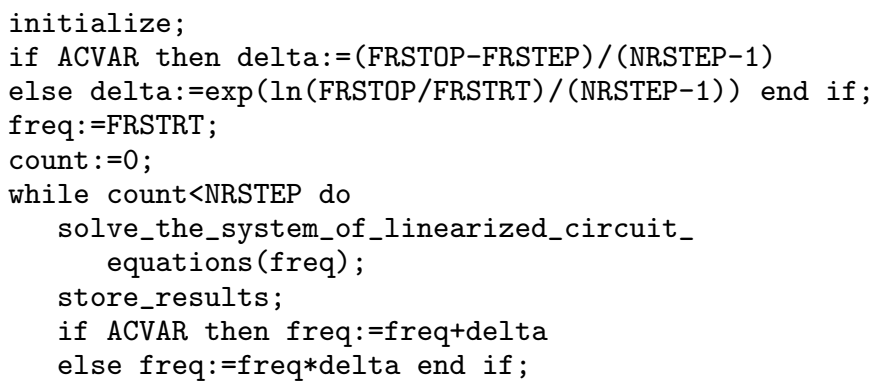


count : $=$ count +1

end while;

The modified version uses a logical variable ACLIST which is TRUE if the analysis is table-driven, otherwise it is FALSE; FRTAB is an array that stores the list of values for a table-driven analysis:

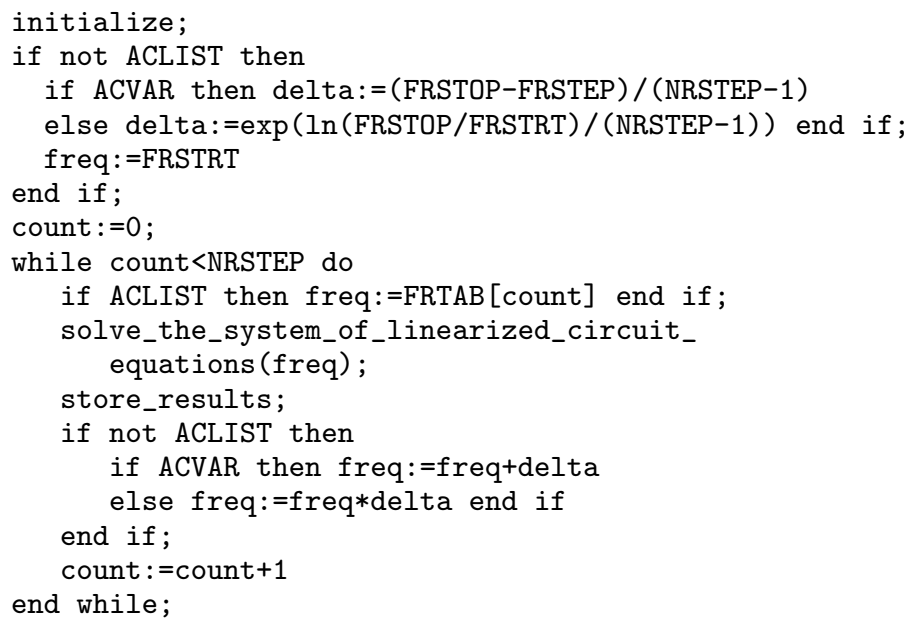

\section{EXAMPLE}

The following example illustrates table-driven DC analysis used for characterization of the transfer curve of a MOSFET inverter:

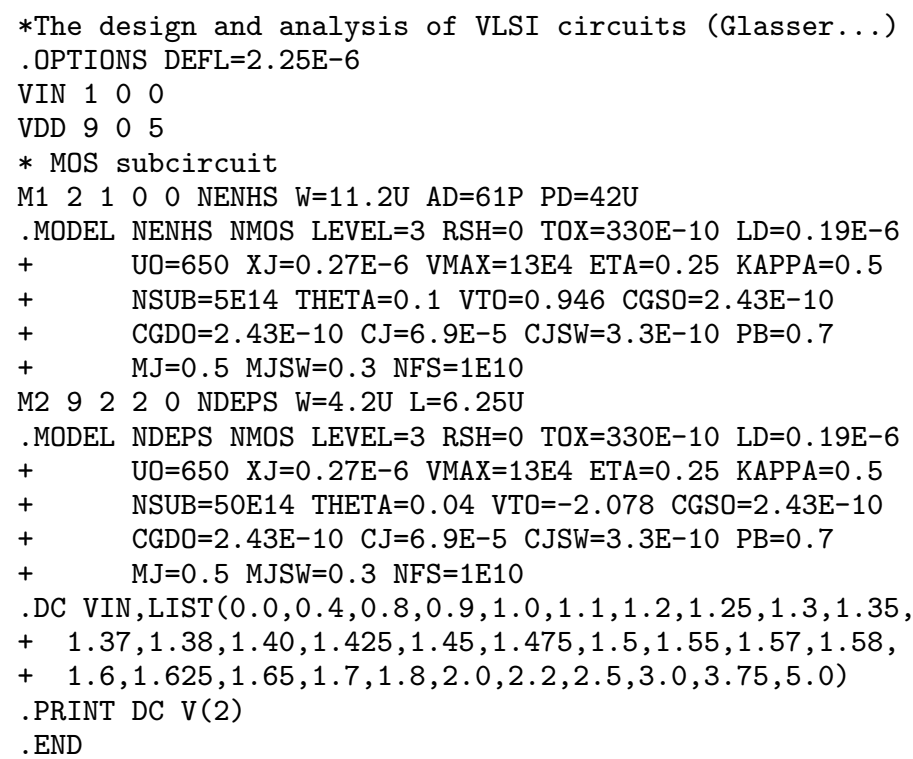

Fig.1 shows the transfer curve of this inverter and the points used in table-driven DC analysis.

The results of this analysis are used in a table-driven controlled source that models the behavior of the inverter [ZZ], and compares the "reference" MOSFET results with two table-driven controlled sources modeling the inverter, (i) E1 that uses linear interpolation, and E2 that uses quadratic interpolation:

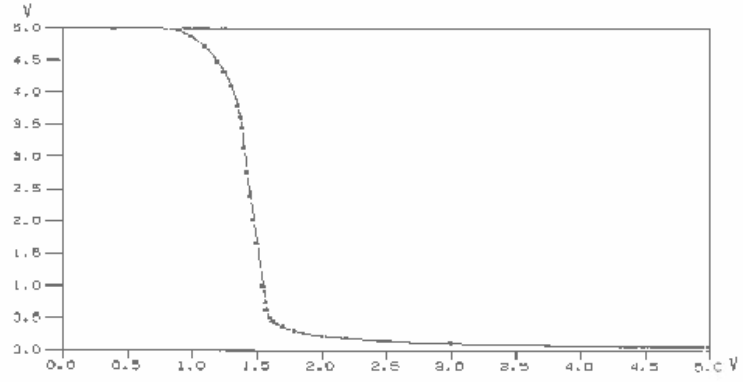

Fig.1. MOS inverter's transfer curve.

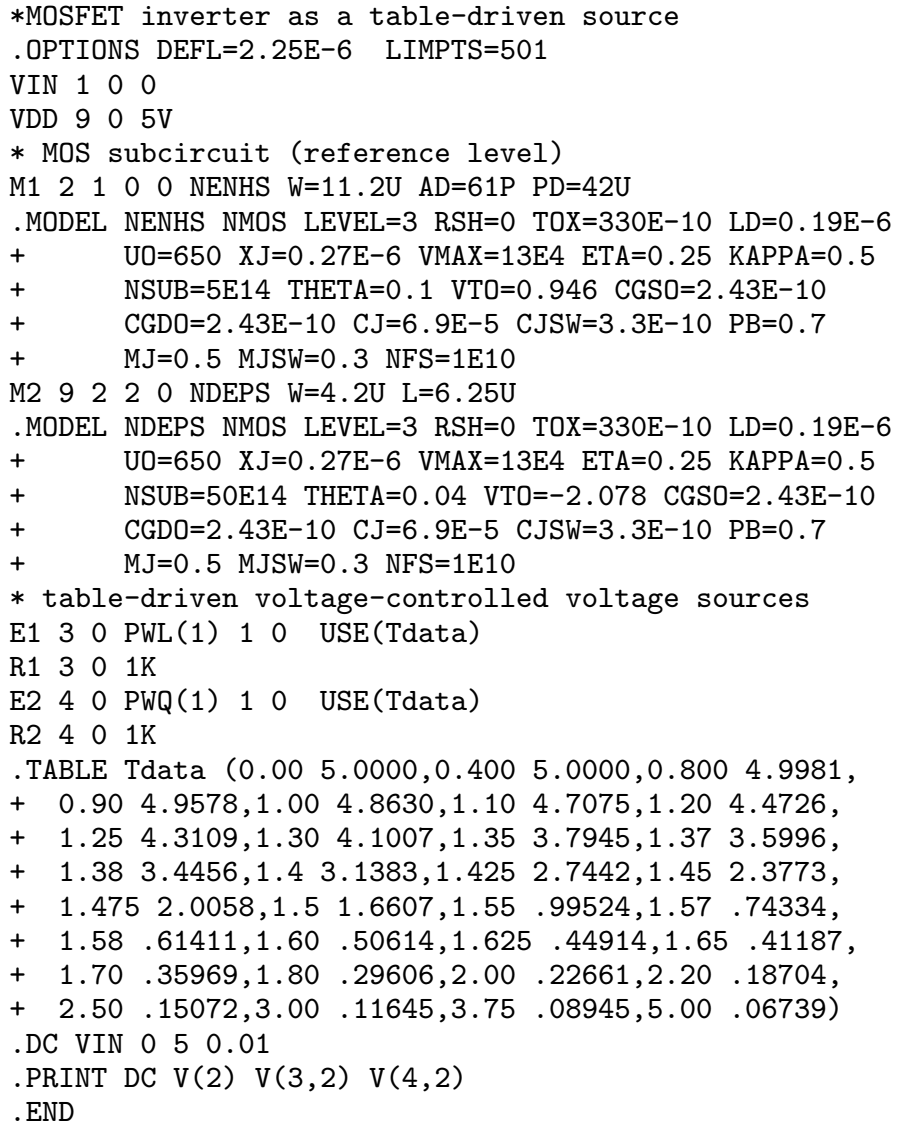

The results of these comparisons, i.e., the differences between the original MOSFET inverter transfer characteristic and the table-driven models, are shown in Fig.2 and Fig.3, for linear and quadratic interpolation, respectively.

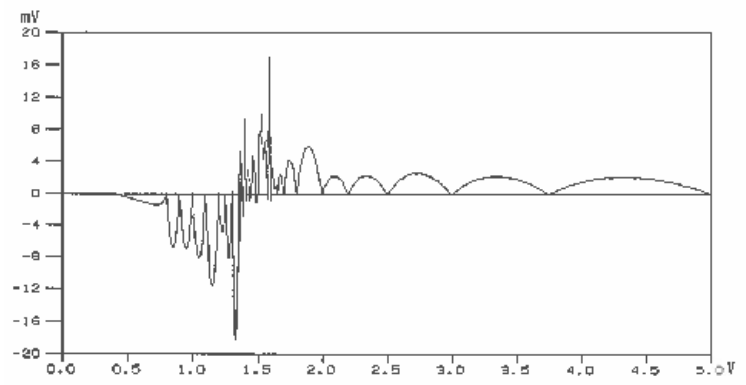

Fig.2. Approximation error for linear interpolation. 


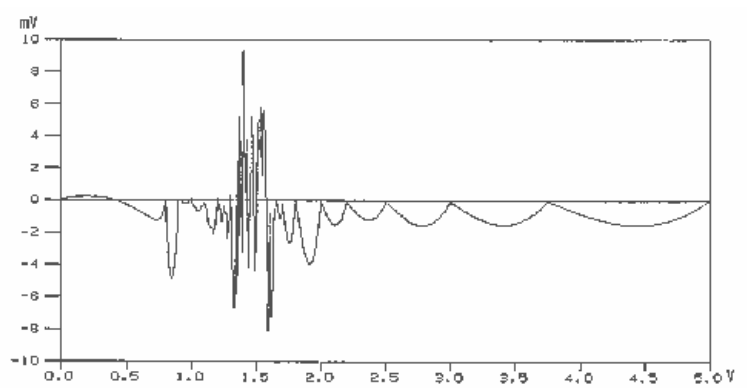

Fig.3. Approximation error for quadratic interpolation.

The approximation errors are at the level of 1 percent or less, and for quadratic interpolation they are approximately two times smaller than for linear interpolation.

\section{CONCLUDING REMARKS}

Uniformly distributed simulation results, typical for SPICE-like simulators, are inadequate for applications, in which the values of independent variables are arbitrarily distributed. Examples of such applications include simulation-based parameter extraction and higherlevel simulation. In parameter extraction, measurement data determine the values of independent variables (voltages, currents, time or frequency) for which the simulation results are needed, so the simulator must be flexible enough to accept arbitrarily distributed measurement data. In higher-level simulation, characteristics of functional blocks are represented by (multidimensional) datadriven elements, in which the accuracy of representation determines the distributions of data points, and the simulators should be flexible enough to deal with such requirements. Table-driven analyses provide simulation flexibility that is required in these applications.

The simple example of MOS inverter modeling shows that data-driven elements can provide accuracy needed in practical applications, at the same time reducing significantly the computational effort required for evaluation of complex models of semiconductor devices [BVS,Rau].

\section{Acknowledgement}

The Natural Sciences and Engineering Research Council of Canada partially supported this research through Operating Grant A8222.

\section{$R$ e f e r e n c e s}

[BVS] J. Barby, J. Vlach, K. Singhal, "Optimized polynomial splines for FET models" Proc. 1984 Int. Symp. on Circuits and Systems, Montreal, Canada, pp.11591162.

[Coh] E. Cohen, "Program reference for SPICE 2"; Memorandum UCB/ERL M592, University of California, Berkeley, CA 94720, 1976.
[GD] L.A. Glasser, D.W. Dobberpuhl, "The design and analysis of VLSI circuits"; Addison-Wesley 1985.

[Rau] K-G. Rauch, "A table model for circuit simulation"; Proc. ESSCIRC '86, pp.211-213, 1986.

[Vlad] A. Vladimirescu, K. Zhang, A.R. Newton, D.O. Pederson, A.L. Sangiovanni-Vincentelli, "SPICE Version 2G - User's Guide"; Department of Electrical Engineering and Computer Sciences, University of California, Berkeley, CA 94720, 1981.

[Z1] W.M. Zuberek, "SPICE-PAC version 2G6c - an overview"; Technical Report \#8903, Department of Computer Science, Memorial University of Newfoundland, St. John's, Canada A1C-5S7, 1989.

[Z2] W.M. Zuberek, "SPICE-PAC version 2G6c - user's guide"; Technical Report \#8902, Department of Computer Science, Memorial University of Newfoundland, St. John's, Canada A1C-5S7, 1989.

[ZK] W.M. Zuberek, A. Konczykowska, "FIT-2, an extraction program based on the SPICE-PAC simulation software"; Proc. 34th Midwest Symp. on Circuits and Systems, Monterey CA, pp.142-145, 1991,

[ZZ] M.S. Zuberek, W.M. Zuberek, "Enhanced controlled sources as device models in the SPICE-PAC simulation package"; Proc. 30th Midwest Symp. on Circuits and Systems, Syracuse NY, pp.603-606, NorthHolland 1988. 